Pacific Journal of Mathematics

TOPOLOGICAL SEMILATTICES ON THE TWO-CELL 


\section{TOPOLOGICAL SEMILATTICES ON THE TWO-CELL}

To Professor A. D. Wallace on his 60th birthday

\section{DENNISON R. BROWN}

Topological lattices on the n-cell have been studied by L.W. Anderson, A.D. Wallace, A.L. Shields, and L.E. Ward, Jr. In particular, these authors have papers setting forth conditions under which a topological lattice on the two-cell is topologically isomorphic to the product lattice $I \times I$.

The primary purpose of this paper is the investigation of topological semilattices (commutative, idempotent topological semigroups) on the two-cell which retain the lattice like property that for each element $x,\{y: x \leqq y\}$ is a connected set. Specifically, it is shown that any such entity is the continuous homomorphic image of one of a fixed pair of semilattices on the two-cell, where the choice of domain depends on the location of the zero element.

It is also proved that a TSL on the two-cell has an identity (a unique maximal element) and $\{y: x \leqq y\}$ connected for each element $x$ if and only if it is the continuous homomorphic image of $I \times I$. Also, if $\{y: x \leqq y\}$ is connected for each element $x$, then $S$, a TSL on the two-cell, is generated by its boundary $B$ in the sense that $B^{2}=S$.

Semilattices on the $n$-cell are also discussed. Let $S$ be such an object with boundary $B$. It is proved that if $x$ is a maximal element of $S$, then $x \in B$. If $S$ has an identity, 1, and $T$ is a continuum chain from 1 to 0 , then $S=B T$.

Finally, let $S$ be a continuum TSL with 1 and let $A$ be the subset defined by $x \in A$ if and only if $\{y: x \leqq y\}$ is connected. Then (1) $x \in A$ if and only if there is a continuum chain from 1 to $x ; \operatorname{and}(2) A$ is a nondegenerate continuum sub-TSL of $S$.

Topological lattices on the $n$-cell have been studied in [1], [6], and in [8]. In particular, these papers set forth conditions under which a topological lattice on the two-cell is iseomorphic (topologically isomorphic) to the product lattice $I \times I$.

The primary purpose of this paper is the investigation of topological semilattices (commutative, idempotent topological semigroups) on the two-cell which retain the lattice-like property that for each element $x, M(x)$ is a connected set (see below). Specifically, we show that any such entity is the continuous homomorphic image of one of a fixed pair of semilattices, where the choice of domain depends upon the location of the zero.

Received March 12, 1964. This work was supported in part by NSF contract GP-1637. 
Section 3 discusses semilattices on the $n$-cell. The role of the boundary sphere in determining the multiplication is seen to be quite important.

The next section is the main body of the paper. In addition to the theorems indicated above, we prove that any topological semilattice on the two-cell which has a unique maximal element and all $M(x)$ connected must be the continuous homomorphic image of $I \times I$. In particular, any topological lattice has these properties. We also show that, if each $M(x)$ is connected, then a topological semilattice $S$ on the two-cell is generated by its boundary $B$ in the sense that $B^{2}=S$.

In $\S 5$ we prove that if $S$ is a compact, connected topological semilattice with identity, then the subset of elements $x$ such that $M(x)$ is connected is a compact connected subsemilattice of $S$.

We are indebted to Professors H. Cohen and R.J. Koch for their helpful comments and support.

2. Preliminaries. A topological semilattice (hereafter TSL) is a pair $(S, \leqq)$ such that $S$ is a Hausdorff topological space, $\leqq$ is a continuous semilattice ordering on $S$. Equivalently, $S$ is a commutative, idempotent topological semigroup with $x \leqq y$ if and only if $x y=x$.

An element $x$ of $S$ is maximal if it is dominated by no other element of $S$; that is, $x y=x$ implies $y=x$. A minimal element is defined dually. It is well known that a compact TSL has maximal elements and a unique minimal element. For $x \in S$, let $M(x)=$ $\{y: x \leqq y\}, L(x)=\{y: y \leqq x\}$. It is easy to verify that $L(x)=S x$. These are closed subsemilattices of $S$ [10].

A chain is a totally ordered subset of $S$. Of primary interest here are compact, connected chains; in case $S$ is metric these are known to be arcs [13] and will be referred to henceforth as arc chains.

The following theorem, due to Koch [4], is stated without proof.

THEOREM A. If $S$ is a compxct, connected, metric TSL with zero (0), then every $x \in S$ is connected to 0 by an arc chain.

If $S$ is as stated in Theorem $\mathrm{A}$ and has also $M(x)$ connected for each $x \in S$, then, by replacing $S$ by $M(x)$ in the theorem, is may be seen that any pair of comparable elements in $S$ is connected by an arc chain.

A space $S$ is homotopically trivial if $\pi_{i}(S)=0, i>0$ where $\pi_{i}(S)$ is the $i$ th homotopy group of $S$. The following result extends slightly a theorem of Anderson and Ward [2].

THEOREM B. If $S$ is an arcwise connected idempotent semigroup, 
with (0), then $S$ is homotopically trivial.

Proof. Let $f: I_{1} \times \cdots \times I_{n} \rightarrow S$ with $f($ Bndry $(I \times \cdots \times I)=0$. Define $g:\left(I_{1} \times \cdots \times I_{n}\right) \times I \rightarrow S$ by

$$
g\left(x_{1}, \cdots, x_{n}, r\right)=f\left(x_{1}, \cdots, x_{n}\right) f\left(x_{1}-x_{1} r, \cdots, x_{n}-x_{n} r\right) .
$$

Henceforth, the letter $I$ will be reserved to represent the TSL on the arc $[0,1]$ defined by $x y=\min (x, y)$, where the ordering is that inherited from the real numbers. Any arc chain is iseomorphic to $I[5]$.

3. Semilattices on the $n$-cell. Throughout this section, $S$ represents a TSL whose underlying space is an $n$-cell, and $B$ the boundary $n-1$ sphere of $S$. If $S$ has an identity, 1 , then 1 is clearly the unique maximal element of $S$. It is well-known [7] that $1 \in B$. The following order-theoretic version of the maximum modulus theorem generalizes this statement.

Theorem 1. Let $x$ be a maximal element of $S$. Then $x \in B$.

Proof. By the maximality of $x$, and Theorem B, $S \backslash\{x\}$ is a subsemilattice, homotopically trivial. Hence $x \notin S \backslash B$.

In [3] and in [5] it was shown that, under certain conditions, the multiplication in $S$ is determined by that in $B$ together with that in a certain arc subsemigroup. The next theorem is of a similar nature.

Theorem 2. Let $S$ have a 1 , and let $T$ be any arc chain from 1 to 0 . Then $S=B T$.

Proof. Since $B \subset B T$, it suffices to show $B T$ is contractible. Since $T$ is an interval, define $g:(B T) \times T \rightarrow B T$ by $g(b t, r)=b t r$. Then $g(b t, 1)=b t$, and $g(b t, 0)=0$. The function $g$ is clearly continuous, hence the proof is complete.

4. Semilattices on the 2-cell. The following lemmas will be useful in the sequel.

LEMMA 1. Let $S$ be a topological semilattice in which, for each $x, M(x)$ is a connected set. Let $f: S \rightarrow T$ be a continuous homomorphism of $S$ onto $T$. Then, for each $y \in T, M(y)$ is connected; furthermore $f$ is a monotone map. 
Proof. The continuous homomorphic image of an arc chain is clearly an arc chain, although possibly degenerate. Let $y \in T, z \in M(y), y=$ $f(a), z=f(b)$. Then $y=y z=f(a) f(b)=f(a b)$. Let $C$ be an arc chain in $S$ from $b$ to $a b$. Then $f(C)$ is again such from $z$ to $y$.

Now let $X=f^{-1}(y), a, b \in X$. Let $C, D$ be arc chains from $a$ to. $a b, b$ to $a b$ respectively. Then $C \cup D \subseteq X$, hence $X$ is connected.

Lemma 2. Let $S$ be a two cell, $S=C \cup D, C \cap D=\square$, where $C$ and $D$ are arc wise connected sets. Let $B$ be the boundary of $S$ and suppose $B \cap C \neq \square \neq B \cap D$. Then $C \cap B$ and $D \cap B$ are each connected sets.

Proof. If $C \cap B$ is not connected, then clearly neither is $D \cap B$. Choose $a, b$ from different components of $C \cap B$, and let $T$ be an arc in $C$ connecting $a, b$. Choose $d, e$ from different components of $D \cap B$, so that $\{a, b\}$ separates $d$ and $e$ in $B$, and let $J$ be an arc in $D$ connecting $d, e$. Then $J$ and $T$ cannot be disjoint [13], which contradicts $C \cap D=\square$.

In the remainder of this section, $S$ will represent a TSL whose underlying space is a two-cell, and $B$ the boundary circle of $S$.

Corollary. Let $a, b \in B$, with $M(a b) a$ connected set. Decompose $B$ into arcs $P, Q$ with $P \cap Q=\{a, b\}$. Then either $P \cong M(a b)$ or $Q \subseteq M(a b)$.

Proof. $M(a b)$ is arcwise connected by Theorem A. On the other hand, any $x \in S \backslash M(a b)$ can be connected to 0 by an arc chain $T$ in $S$. Clearly $T \cap M(a b)=\square$. Hence $S \backslash M(a b)$ is arcwise connected. By the lemma above, $M(a b) \cap B$ is therefore connected and the result follows.

Methods used in portions of the proof of the following theorem are similar to those used in [8].

THEOREM 3. Suppose $S$ has a 1. These are equivalent:

(i) for each $x, M(x)$ is a connected set;

(ii) $B$ is the union of two maximal arc chains of $S$;

(iii) $S$ is the continuous homomorphic image of $I \times I$.

Proof. (i) implies (ii). Fix $a \in B, a \neq 1$. By the above corollary, $M(a)$ must contain one of the boundary arcs between $a$ and 1. Designate this arc by $Q$ and let $p \in Q, p \neq a$. Let $P$ be the boundary arc between $p$ and 1 which is contained in $Q$. Then $P \subseteq M(p)$, for if not then $a \in M(p)$, which is false. It follows that any element of $Q$ 
compares with $p$, and hence $Q$ is totally ordered. Let $J$ be a maximal chain in $B$, with $a, 1 \in J$. By continuity of multiplication, $J$ is closed and therefore proper in $B$. Let $t \in B \backslash J$, and let $K$ be a maximal chain in $B$, with $t, 1 \in K$. By the maximality of $J$ and $K, B=$ $J \cup K$. From the anti-symmetry of the relation ( $\leqq$ ), $J$ and $K$ have the same minimal element, $z$. Now $M(z)$ is a compact, connected semilattice, homotopically trivial by Theorems $\mathrm{A}$ and $\mathrm{B}$. Since $B \subseteq M(z)$, $M(z)=S$, and hence $z=0$. The arc chains $J, K$ are thus maximal in $B$. If $c \in S \backslash B$, then $M(c) \cap J$ and $L(c) \cap J$ are closed, disjoint subsets of $J$, and hence fail to exhaust $J$. Then maximality of $J, K$ in $S$ is now immediate.

(ii) implies (iii). Let $R$ be the closed ideal $(I \times\{0\}) \cup(\{0\} \times I)$ of $I \times I$; and let $M$ be the Rees quotient $(I \times I) / R$. This TSL on the 2 -cell has the properties that every nonzero element of $M$ is represented uniquely as a product of two boundary elements, one from each of the maximal chains composing the boundary of $M$ (if $a \in C$, then $a=a \cdot 1)$, and $a b=0$ implies either $a=0$ or $b=0$. Denote the boundary of $M$ by $C=V \cup W$, and the boundary of $S$ by $B=J \cup K$, with $V, W, J, K$ maximal arc chains.

Let $f_{1}: V \rightarrow J$ and $f_{2}: W \rightarrow K$ be iseomorphisms. Define $f^{*}: M \rightarrow S$ by $f^{*}(x)=f_{1}(a) f_{2}(b)$, where $a b=x, a \in V, b \in W$. The only element of $M$ which has a nonunique representation in this manner is 0 ; but $a b=0$ requires that one of $a, b=0$. Hence $f^{*}$ is well defined, and the following diagram is commutative:

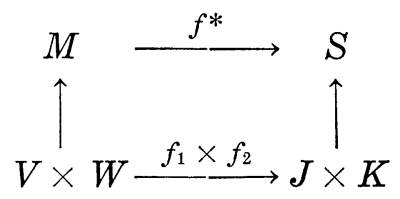

Here, the vertical arrows represent the respective multiplication functions. Since these functions, together with $f_{1}$ and $f_{2}$, are continuous, and $V$ and $W$ are compact, it follows that $f^{*}$ is continuous.

Next, let $a=v_{1} w_{1}, b=v_{2} w_{2}$ be elements of $M$. Then

$$
\begin{aligned}
f^{*}(a b) & =f^{*}\left(v_{1} w_{1} v_{2} w_{2}\right)=f^{*}\left(\left(v_{1} v_{2}\right)\left(w_{1} w_{2}\right)\right)=f_{1}\left(v_{1} v_{2}\right) f_{2}\left(w_{1} w_{2}\right) \\
& =f_{1}\left(v_{1}\right) f_{1}\left(v_{2}\right) f_{2}\left(w_{1}\right) f_{2}\left(w_{2}\right)=f^{*}\left(v_{1} w_{1}\right) f^{*}\left(v_{2} w_{2}\right)=f^{*}(a) f^{*}(b) .
\end{aligned}
$$

Hence $f^{*}$ is a homomorphism.

Finally, $f^{*}(M)$ is a compact connected TSL containing $B$; by Theorems $\mathrm{A}$ and $\mathrm{B}$ it follows that $f^{*}(M)=S$. The natural map of $I \times I$ onto $(I \times I) / R$ is now composed with $f^{*}$ to obtain the desired result.

(iii) implies (i). Clearly $I \times I$ has $M(x)$ connected for each $x$. By Lemma $1, S$ has this property also. 
CoRollary 3.1. If $S$ has $a 1$ and $M(x)$ connected for each $x \in S$, then $0 \in B$.

CoRollary 3.2. If $S$ is a topological lattice on the 2-cell, then $S$ is the continuous (semilattice) homomorphic image of $I \times I$.

Proof. As a topological lattice, $S$ has a 1 and has $M(x)$ connected for each $x \in S$.

THEOREM 4. If $M(x)$ is connected for each $x \in S$, then $B^{2}=S$.

Proof. Suppose $0 \in B$. It will first be shown that $B^{3} \subseteq B^{2}$. Let $a, b, c \in B$. In order to prove that $a b c \in B^{2}$, it suffices to assume that $a, b, c$ are distinct and nonzero. Assume also that these points are named so that $0, b$ are in different components of $B \backslash\{a, c\}$. By the corollary to Lemma 2 , one component of $B \backslash\{a, c\}$ lies in $M(a c)$. Since $0 \notin M(a c)$, it follows that $b \in M(a c)$, hence $a b c=a c$ and $B^{3} \cong B^{2}$. Hence $B^{4}=B^{3} B \subseteq B^{2} B \subseteq B^{2}$. Since $B^{2}$ is a compact connected TSL, and $B \subseteq B^{2}$, it follows from Theorems $\mathrm{A}$ and $\mathrm{B}$ that $B^{2}=S$.

Now suppose $0 \in S \backslash B$, and again select $a, b, c$ distinct elements of $B$. If any of $a b, a c, b c, a b c \in B$, then immediately $a b c \in B^{2}$. Similarly, it may be assumed that $a \notin M(b c), b \notin M(a c), \quad c \notin M(a b)$. By the corollary to Lemma 2, it follows that $B=M(a b) \cup M(a c) \cup M(b c)$. But the latter subset is included in $M(a b c)$. Since $M(a b c)$ is a compact, connected TSL and $B \subseteq M(a b c)$, it again follows that $M(a b c)=S$, and therefore $a b c=0$. It has now been shown that $B^{3}=B^{2} \cup\{0\}$; thus $B^{2} \cup\{0\}$ is compact and connected. Furthermore, $\left(B^{2} \cup\{0\}\right)^{2} \subseteq B^{4} \cup\{0\} \subseteq B^{3} \cup\{0\}$; hence $B^{2} \cup\{0\}$ is a subsemilattice containing $B$. This yields $B^{2} \cup\{0\}=S$. But $B^{2}$ is compact, hence $0 \in B^{2}$. Consequently $B^{3} \subseteq B^{2}$, and as before, $B^{2}=S$.

The next pair of theorems shows that the structure of $S$ when $0 \in B$ is essentially different from that occurring when $0 \in S \backslash B$. Let $T=\{(x, y) \in I \times I: x+y \leqq 1\}$. Note $T$ is a subsemilattice of $I \times I$.

THeOREM 5. The semilattice $S$ has $0 \in B$ and $M(x)$ connected for each $x$ if and only if $S$ is a continuous homomorphic image of $T$.

Proof. As in Theorem 3, let $R=(I \times\{0\}) \cup(\{0\}) \times I)$. Let $N=$ $T / R$, the Rees quotient of $T$. Let $D$ be the boundary circle of $N$. Note that every nonzero element of $N$ has a unique representation as a product of two not necessarily distinct elements of $D$, and that $a b=0$ implies $a=0$ or $b=0$. Now let $f: D \rightarrow B$ homeomorphically, with $f(0)=0$, and extend $f$ to $f^{*}: N \rightarrow S$ by $f^{*}(c)=f(a) f(b)$, where $a, b \in D$. As in Theorem $3, f^{*}$ is well defined, and the following diagram is commutative : 


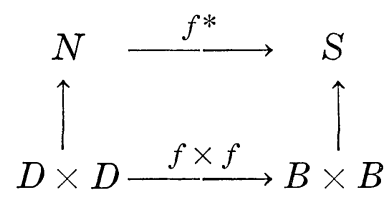

Vertical arrows represent the respective multiplication functions of $N$ and $S$. Since these functions and $f$ are continuous and $D$ is compact, $f^{*}$ is continuous. By Theorem $4, f^{*}$ maps $N$ onto $S$.

It remains to show that $f^{*}$ is a homomorphism. To this end, let $c=a b, a=p q, b=r s$, with $p, q, r, s$ distinct nonzero elements of $D$. Some unique pair of $p, q, r, s$ separates the remaining pair from 0 on $D$; suppose $p, r$ and 0 lie in different components of $D \backslash\{q, s\}$. By the corollary to Lemma 2, $\{p, r\} \subseteq M(q s)$. Hence $a b=p q r s=q s$, and $f^{*}(c)=f^{*}(a b)=f^{*}(q s)=f(q) f(s)$. On the other hand, since $f$ is a homeomorphism on $D$, it follows that $\{f(p), f(r)\}$ and $\{f(0)=0\}$ lie in different components of $B \backslash\{f(q), f(s)\}$. Hence $\{f(p), f(r)\} \subseteq M(f(q) f(s))$ and therefore $f(q) f(s)=f(p) f(q) f(r) f(s)=f^{*}(p q) f^{*}(r s)=f^{*}(\alpha) f^{*}(b)$. The argument is similar if $p, q, r, s$ occur in a different order in $D$. This portion of the proof is now complete.

The converse follows from the fact that $T$ has $M(x)$ connected for each $x$ and Lemma 1 , together with the fact that a monotone map of a a two-cell onto a two-cell must take boundary onto boundary [12].

Now, let $W$ be the disk of radius one, centered at the origin of a plane, and let $F$ be the boundary circle of $W$. If $x, y \in F$, let $x y$ be the midpoint of the chord joining $x$ and $y$. This is transparently continuous, and note that a nonzero point of $W$ is uniquely represented as the product of two boundary points. To extend the multiplication to all of $W$ let $\alpha=w x, b=y z$ where $a, b \in W$ and $w, x, y, z \in F$; set $a b=0$ if each boundary arc containing $w, x, y$ and $z$ has length $\geqq \pi$, and otherwise let $a b$ be the product of those two of the four elements $w, x, y$ and $z$ whose distance apart is a maximum. Again continuity is obvious, as is the fact that multiplication is commutative and idempotent.

It is certainly desirable to give an alternative, order-theoretic description of $W$. For each $x \in W$ let $L(x)$ be the intersection of all circular disks which are tangent to $F$, which contain $x$, and whose boundaries contain 0 . This is a semilattice partial order and $L(x) \cap L(y)$ is precisely $L(z)$ for that $z(=x y) \in L(x) \cap L(y)$ which is at maximum distance from 0 . In this manner, it if easily seen that $M(x)$ is connected for each $x \in W$. Note that, if $a, b, c, d \in F$, then $a b c d \neq 0$ if and only if one of these elements, say $a$, has the property that $b, c, d$ lie in the same component of $F \backslash\{a,-a\}$, where $(-a)$ represents the

1 The author is indebted to the referee for improving the description of the semigroup $W$. 
point of $F$ antipodal to $a .^{(1)}$

Theorem 6. Let $0 \in S \backslash B$. Then $M(x)$ is a connected set for $\epsilon$ ach $x \in S$ if and only if $S$ is the continuous homomorphic image of $W$.

Proof. One implication is an immediate consequence of Lemma 1, since $W$ has $M(x)$ connected for all $x$. Therefore, assume that $S$ has $M(x)$ connected for all $x, 0 \notin B$. It will be shown that $S$ is the continuous homomorphic image of $W$.

First, suppose that $f$ is a continuous map of $F$ onto $B$. Define $f^{*}: W \rightarrow S$ by $f^{*}(x)=f(a) f(b)$, where $x=a b, a, b \in F$. Recall that, if $x \neq 0$, then this representation of $x$ is unique, and $f^{*}(x)$ is therefore well defined. On the other hand 0 may be expressed only as the product of any pair of antipodal elements of $F$. Hence, in order that $f^{*}$ be well defined, $a b=0, a, b \in F$ must imply $f(a) f(b)=0$ in $S$. The construction of a continuous map $f$ with this property is the major portion the proof. For $x \in B$, define $A(x)=\{y \in B: x y=0\}$. In the sequel, the expression $[a, b], a, b \in B$, will represent the counterclockwise arc of $B$ from $a$ to $b$.

(A) For each $x \in B, A(x)$ is a continuum; further, there exist $y, z \in B$ such that $y z=0, x \in[y, z]$ and $A(x) \cap[z, y]$ is nonempty. By Theorem $4, B^{2}=S$; hence there exist $y, z \in B$ such that $y z=0$. Let $x \in[y, z], t \in[z, y]$. By the corollary to Lemma 2, then either $y \in M(x t)$ or $z \in M(x t)$. Hence $x[z, y] \subseteq L(y) \cup L(z)$. Since $x[z, y]$ is connected and $L(y) \cap L(z)=\{0\}$, it follows that for some $t \in[z, y]$, $x t=0$; hence $A(x) \neq \square$. Next, let $a, b \in A(x), \quad x \in[a, b], t \in[b, a]$. Again by the corollary to Lemma 2, either $a \in M(x t)$ or $b \in M(x t)$. Say $a \in M(x t)$; then $x t=a(x t)=(a x) t=0$. Therefore $[b, a] \subseteq A(x)$; by using the compactness of $B$ to obtain a maximal interval, it may be seen that $A(x)$ is an (possibly degenerate) arc in $B$.

(B) There exist $a_{0}, a_{1} \in B$ such that $a_{0} a_{1}=0$ and for every $x \in\left(a_{0}, a_{1}\right), A(x) \subseteq\left(a_{1}, a_{0}\right)$. Let $a \in B$; there exists $a_{1} \in A(a)$ such that $\left[a, a_{1}\right] \cap A(a)=\left\{a_{1}\right\}$ and there exists $a_{0} \in A\left(a_{1}\right)$ such that $\left[a_{0}, a_{1}\right] \cap A\left(a_{1}\right)=$ $\left\{a_{0}\right\}$. Then $a_{0} a_{1}=0$ and we observe that $a \in\left[a_{1}, a_{0}\right]$. It $x \in\left(a_{0}, a_{1}\right)$ then it is obvious that $a_{1} x \neq 0 \neq a x$. Now by $(A), A(x)$ meets $\left[a_{1}, a\right]$ and, since it is connected, $A(x) \subset\left(a_{1}, a\right) \subset\left(a_{1}, a_{0}\right)$.

(C) Let $a, b, c, d$, e be five elements of $B$ occurring in counter clockwise order as listed; suppose also that $a c=0=b d$ and that $b e \neq 0 \neq c e$. Then $A(e) \subseteq(b, c)$. For, from $a c=0, e c \neq 0, e \in(c, a)$, we have $A(e) \subset(e, c)$ by (A). Similarly, from $b d=0, e b \neq 0, e \in(d, b)$, we have $A(e) \subset(b, e)$. Therefore $A(e) \subset(e, c) \cap(d, b)=(b, c)$. 
The function $f: F \rightarrow B$ will now be defined.

(D) Choose elements $a_{0}, a_{1}$ of $B$ as in part $B$ above. For convenience, the antipodal point of $x \in F$ is denoted by $-x$. Fix any $x \in F$ and define $f(x)=a_{0} . f(-x)=a_{1} . F$ is now decomposed into the two closed intervals $[x,-x]$ and $[-x, x]$, while $B=\left[a_{0}, a_{1}\right] \cup\left[a_{1}, a_{0}\right]$. The scheme is now as follows: $f$ will map a dense subset of $[x,-x]$ onto a dense subset of $\left[a_{0}, a_{1}\right]$, and a dense subset of $[-x, x]$ onto a dense subset of $\left[a_{1}, a_{0}\right]$ in an order preserving manner; furthermore $f(y) f(-y)$ will be 0 for every $y$ in either the dense subset of $[x,-x]$ or the dense subset of $[-x, x]$. The function $f$ will then be extended through standard methods into a continuous map of $F$ onto $B$; that $f(y) f(-y)=$ 0 for all $y \in F$ will be a consequence of this method of construction.

For ease of notation, set $-x=x_{1}=y_{0}, x_{0}=x=y_{1}$ in $F$ and $a_{1}=b_{0}$, $b_{1}=a_{0}$ in $B$. Now $F=\left[x_{0}, x_{1}\right] \cup\left[y_{0}, y_{1}\right], B=\left[a_{0}, a_{1}\right] \cup\left[b_{0}, b_{1}\right]$. Let $a_{01}$ be the mid-point of $\left[a_{0}, a_{1}\right], x_{01}$ the mid-point of $\left[x_{0}, x_{1}\right], y_{01}\left(=-x_{01}\right)$ that of $\left[y_{0}, y_{1}\right]$. Define $f\left(x_{01}\right)=a_{01}$. By part B), $A\left(a_{01}\right) \subseteq\left(b_{0}, b_{1}\right)$; let $b_{01} \in A\left(a_{01}\right)$ and define $f\left(y_{01}\right)=b_{01}$.

(E) Next, let $b_{001}$ be the mid-point of $\left[b_{0}, b_{01}\right], y_{001}$ that of $\left[y_{0}, y_{01}\right]$, and define $f\left(y_{001}\right)=b_{001}$. Let $x_{001}=-y_{001}$. It is necessary to map $x_{001}$ into some point $a_{001}$ of the interval $\left[a_{0}, a_{01}\right]$. To this end, suppose that $a_{0} b_{001}=0=a_{01} b_{001}$. Then by (A) above $\left[a_{0}, a_{01}\right] \subseteq A\left(b_{001}\right)$. In this case choose $a_{001}$ to be the mid-point of $\left[a_{0}, a_{01}\right]$. If, on the other hand, $a_{0} b_{001}=$ $0 \neq a_{01} b_{001}$, then let $a_{001}=a_{0}$; if $a_{0} b_{001} \neq 0=a_{01} b_{001}$, let $a_{001}=a_{01}$. Finally, if $a_{0} b_{001} \neq 0 \neq a_{01} b_{001}$, then, on applying (C) with $a=b_{01}, b=a_{0}, c=a_{01}$, $d=b_{0}, e=b_{001}$, it follows that $A\left(b_{001}\right) \cong\left(a_{0}, a_{01}\right)$. In this case, choose $a_{001}$ arbitrarily in $A\left(b_{001}\right)$. Similarly, let $b_{011}$ be the mid-point of $\left[b_{01}, b_{1}\right]$; by an argument similar to the one above, there exists $a_{011} \in\left[a_{01}, a_{1}\right]$ such that $a_{011} b_{011}=0$. Choose the appropriate $y_{011}, x_{011}$ in $F$ and define $f\left(x_{011}\right)=$ $a_{011}, f\left(y_{011}\right)=b_{011}$.

(F) In the next stage, mid-points $a_{0001}$ of $\left[a_{0}, a_{001}\right], a_{0011}$ of $\left[a_{001}, a_{01}\right], a_{0101}$ of $\left[a_{01}, a_{011}\right]$ and $a_{0111}$ of $\left[a_{011}, a_{1}\right]$ are chosen as images of the appropriate $x_{i}$. As many as two of the four intervals listed may be degenerate; it is still possible to choose a "mid-point". Suppose, for example, that $a_{001}=a_{0}=a_{0001}$. Then $a_{0001} \cdot b_{001}=0=a_{0001} \cdot b_{0}$, hence by (E) above, $b_{0001}$ may be chosen as the mid-point of $\left[b_{0}, b_{001}\right]$.

(G) At any stage, suppose $a_{i}$ is the mid-point of $\left[a_{j}, a_{k}\right]$. It is then necessary that $b_{i} \in\left[b_{j}, b_{k}\right]$. By examining the products $a_{i} b_{j}$ and $a_{i} b_{k}, b_{i}$ may be chosen precisely by means of the argument used in part (E). A dual argument is obvious in the event that the original choice of mid-point is from a subinterval of $\left[b_{0}, b_{1}\right]$, rather than $\left[a_{0}, a_{1}\right]$. 
(H) By (G) it may be assumed that $f$ has been defined on a dense subset $D$ of $F$ into $B$. The image subset $f(D)$ is dense in $B$, since lengths of complementary intervals clearly approach zero. Also, $f$ is monotonic within $\left[x_{0}, x_{1}\right]$ and $\left[y_{0}, y_{1}\right]$. It is therefore possible to extend $f$ to a continuous map of $F$ onto $W$. Furthermore, choose $x \in F$. It must be shown that $f(x) f(-x)=0$. Let $x \in\left[x_{0}, x_{1}\right]$. It may be assumed that $x \notin D$; let $\left\{x_{i}\right\} \rightarrow x,\left\{x_{i}\right\} \subseteq D$. Then $\left\{-x_{i}\right\} \rightarrow-x$ and $\left\{-x_{i}\right\} \cong D$. By the continuity of $f,\left\{f\left(x_{i}\right)\right\} \rightarrow f(x),\left\{f\left(-x_{i}\right)\right\} \rightarrow f(-x)$. Finally, $\left\{0=f\left(x_{i}\right) f\left(-x_{i}\right)\right\} \rightarrow f(x) f(-x)$, by the continuity of multiplication in $S$. Hence $f(x) f(-x)=0$.

(I) From the discussion prior to (A), the function $f^{*}$ is now well defined from $W$ into $S$, and the following diagram is commutative:

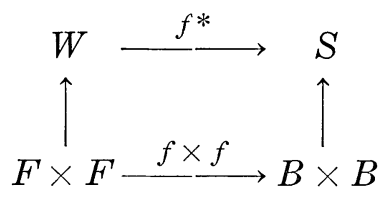

Since $F$ is compact, $f^{*}$ is continuous; by Theorem $4, f^{*}$ maps $W$ onto $S$. It remains to show that $f^{*}$ is a homomorphism. Let $a b=c \in W$, and suppose $a=w x, b=y z$, with $w, x, y, z \in F$. Then $c=w x y z$. If $c \neq 0$, then recall that one of these factors of $c$, say $w$, must have the property that $x, y, z$ are all in the same component of $F \backslash\{w,-w\}$. Suppose further that $\{x, z\} \subseteq[w, y] \subseteq[w,-w]$, where all intervals represented are counter clockwise. Then $c=w y$, hence $f^{*}(c)=f(w) f(y)$. On the other hand, since $f$ is monotone on $[w, y],\{f(x), f(z)\} \subseteq[f(w), f(y)]$ in $B$, and $f(-w) \in[f(y), f(w)]$. If $f(y)=f(-w)$, then $f(w) f(y)=0=$ $f^{*}(c)=f^{*}(a) f^{*}(b)$. If $f(y) \neq f(-w)$, then by the corollary of Lemma 2 , $[f(w), f(y)] \subseteq M(f(w) f(y))$, hence $f^{*}(\alpha) f^{*}(b)=f(w) f(x) f(y) f(z)=$ $f(w) f(y)=f^{*}(c)$. The other cases are handled similarly.

If $a b=c=0$ in $W$, again with $a=w x, b=y z$, then it must be shown that $f(w) f(x) f(y) f(z)=0$. Since $c=0, x, y, z$ cannot all be in the same component of $F \backslash\{w,-w\}$. Suppose $y$ is in one component of $F \backslash\{w,-w\}$, and $\{x, z\}$ in the other. Then, within the component containing $\{x, z\},-y$ must be separated from $-w$ by one of $x, z$; otherwise $w, x, z$, are in the same component of $F \backslash\{y,-y\}$. Suppose $x$ separates $-y$ from $-w$. Then, applying the corollary to Lemma $2,-y \in M(w x)$. Hence $f(-y) \in M(f(w) f(x))$, and therefore

$$
\begin{aligned}
f^{*}(a) f^{*}(b) & =f(w) f(x) f(y) f(z)=[f(-y) f(w) f(x)] f(y) f(z) \\
& =[f(-y) f(y)] f(w) f(x) f(z)=0=f^{*}(c) .
\end{aligned}
$$

The remaining cases are similar. This completes the proof. 
5. Remarks on the general case. It is easy to construct a TSL in which $M(x)$ fails to be connected for some $x$. For example, let $J$ be the arc subsemilattice of $I \times I$ consisting of

$$
(\{0\} \times I) \cup(\{1\} \times I) \cup(I \times\{0\}) .
$$

Then $M((0, y))$ is not connected for any $y>0$. Similarly, the product TSL on the disk $J \times J$ contains points of this nature. For a more complicated example, let $K$ be a subset of $I \times I$ defined as follows: Let a canonical Cantor set $C$ be constructed on the arc $I$, and let $D_{i}$ be the union of the open intervals deleted from $C$ at the $i$ th stage in its construction. Let $K_{i}=\left\{(x, y): x \in D_{i}, \quad\left(3^{i}-1\right) / 3^{i} \leqq y \leqq 1\right\}$. Let $K=(I \times I) \backslash \bigcup_{i} K_{i}$. Then $K$ is a subsemilattice and is topologically a disk. Set $z=(0,1)$. Then $M(z)$ is a Cantor set.

LEMma 3. Let $S$ be any compact connected metric TSL with identity. Let $A=\{x: M(x)$ is a connected subset of $S\}$. Then $x \in A$ if and only if $x$ lies on an arc chain containing 1.

Proof. Suppose $x \in A$. Then $M(x)$ is a compact connected TSL, and by Theorem A, there exists an arc chain $T$ from 1 to $x$. Conversely, let $T$ be an arc chain from 1 to $x$. Clearly $T \subseteq M(x)$. Let $y \in M(x)$. Then $y T$ is connected, and contains $x$ and $y$, and is a subset of $M(x)$. Hence every element of $M(x)$ is connected to $x$ by a connected subset of $M(x)$, and therefore $M(x)$ is connected.

Recall that, if $\left\{A_{n}\right\}_{n} \in \omega$ is a collection of closed subsets of a space $S$, then $\lim \sup \left\{A_{n}\right\}=\{x \in S$; if $x \in U, U$ open in $S, n \in \omega$, then there exists $m>n$ such that $\left.A_{m} \cap U \neq \square\right\}$.

TheOREM 7. Let $S$ and $A$ be as in Lemma 3. Then $A$ is a compact connected subsemilattice of $S$ containing 0 and 1.

Proof. Clearly $0,1 \in A$. Let $x, y \in A$, and let $I, J$ be arc chains from 1 to $x, y$, respectively. Then $I \cup x J$ is an arc chain from 1 to $x y$; by Lemma $3, x y \in A$. Hence $A$ is a subsemilattice. Furthermore, since $I \leqq A$, every element of $A$ lies in a connected subset of $A$ which also includes the element 1 ; hence $A$ is connected. It remains to show that $A$ is closed. Let $\left\{x_{n}\right\}$ be a sequence in $A$, and let $\left\{x_{n}\right\}$ converge to $x$. For each $n$, let $T_{n}$ be an arc chain from 1 to $x_{n}$. Let $T=\lim$ $\sup \left\{T_{n}\right\}$. The set $T$ is known to be connected [12]. To see that $T \subseteq M(x)$, choose $a \in T$, let $\left\{a_{n}\right\}$ cluster to $a, a_{n} \in T_{n}$. Then $\left\{x_{n}\right\}=\left\{a_{n} x_{n}\right\}$ clusters to $a x$, hence $a x=x$. Therefore $a \in M(x)$, and $x$ is connected to 1 inside of $M(x)$. It now follows easily that every element of $M(x)$ lies in a connected set containing $x$ within $M(x)$, hence $x \in A$ and the 
proof is complete.

In all examples on the 2-cell known to the author, $A$ is also locally connected; it is conjectured that if $S$ is locally connected, then $A$ is also. Indeed, it may be that $A$ is a homomorphic retract of $S$.

If $S$ is not assumed to have a 1 , none of the conclusions of the above theorem need hold. In particular, certain subsemilattices of the TSL $J \times J$ mentioned earlier in this section fail in these respects.

\section{BIBLIOGRAPHY}

1. L.W. Anderson, Topological lattices and n-cells, Duke Math. J. 25 (1958), 205-208.

2. L.W. Anderson and L.E. Ward, Jr., A structure theorem for topological lattices, Proceeding of the Glasgow Mathematical Association, Vol. V (1961), 1-3.

3. A.L. Hudson, Some semigroups on the two-cell, Proc. Amer. Math. Soc. 10 (1959), 648-655.

4. R.J. Koch, Arcs in partially ordered spaces, Pacific J. Math. 9 (1959), 723-728.

5. P.S. Mostert and A.L. Shields, On the structure of semigroups on a compact manifold with boundary, Ann. Math. 65 (1957), 117-143.

6. A.L. Shields, The n-cube as a product semigroup, Michigan Math. J. 4 (1957), $165-166$.

7. A.D. Wallace, Cohomology, dimension, and mobs, Summa Brasilia Math. 3 (1953), $43-54$.

8. Factoring a lattice, Proc. Amer. Math. Soc. 9 (1958), 250-252.

9. A theorem on acyclicity, Bull. Amer. Math. Soc. 67 (1961), 123-124.

10. L. E. Ward, Partially ordered topological spaces Proc. Amer. Math. Soc. 5 (1954), 144-161.

11. L.E. Ward, Jr., Concerning Koch's theorem on the existence of arcs, to appear.

12. G.T. Whyburn, Analytic topology, New York, 1942.

13. R.L. Wilder, Topology of manifolds, New York, 1949.

The University of TenNessee 


\section{PACIFIC JOURNAL OF MATHEMATICS}

\section{EDITORS}

H. Samelson

Stanford University

Stanford, California

R. M. Blumenthal

University of Washington

Seattle, Washington 98105
J. Dugundu

University of Southern California Los Angeles, California 90007

*Richard Arens

University of California

Los Angeles, California 90024

\section{ASSOCIATE EDITORS}
E. F. BECKENBACH
B. H. NeumanN
F. WOLF
K. YOSIDA

\section{SUPPORTING INSTITUTIONS}

UNIVERSITY OF BRITISH COLUMBIA CALIFORNIA INSTITUTE OF TECHNOLOGY UNIVERSITY OF CALIFORNIA MONTANA STATE UNIVERSITY

UNIVERSITY OF NEVADA

NEW MEXICO STATE UNIVERSITY

OREGON STATE UNIVERSITY

UNIVERSITY OF OREGON

OSAKA UNIVERSITY

UNIVERSITY OF SOUTHERN CALIFORNIA
STANFORD UNIVERSITY

UNIVERSITY OF TOKYO

UNIVERSITY OF UTAH

WASHINGTON STATE UNIVERSITY

UNIVERSITY OF WASHINGTON

AMERICAN MATHEMATICAL SOCIETY CALIFORNIA RESEARCH CORPORATION SPACE TECHNOLOGY LABORATORIES NAVAL ORDNANCE TEST STATION 


\section{Pacific Journal of Mathematics}

\section{Vol. 15, No. $1 \quad$ September, 1965}

Donald Charles Benson, Unimodular solutions of infinite systems of linear

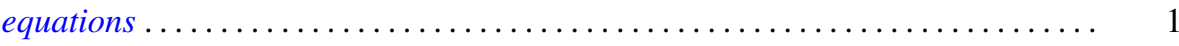

Richard Earl Block, Transitive groups of collineations on certain designs . . . . . . 13

Barry William Boehm, Existence of best rational Tchebycheff approximations .... . 19

Joseph Patrick Brannen, A note on Hausdorff's summation methods . . . . . . . . . . 29

Dennison Robert Brown, Topological semilattices on the two-cell ............ 35

Peter Southcott Bullen, Some inequalities for symmetric means . . . . . . . . . . 47

David Geoffrey Cantor, On arithmetic properties of coefficients of rational

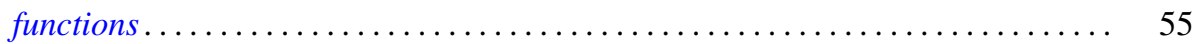

Luther Elic Claborn, Dedekind domains and rings of quotients . . . . . . . . . 59

Allan Clark, Homotopy commutativity and the Moore spectral sequence ........ 65

Allen Devinatz, The asymptotic nature of the solutions of certain linear systems of

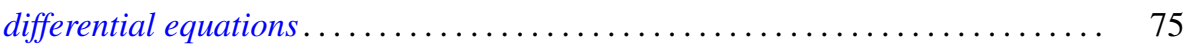

Robert E. Edwards, Approximation by convolutions ................... 85

Theodore William Gamelin, Decomposition theorems for Fredholm operators . . . . . 97

Edmond E. Granirer, On the invariant mean on topological semigroups and on

topological groups .................................. 107

Noel Justin Hicks, Closed vector fields . . . . . . . . . . . . . . . . . . . 141

Charles Ray Hobby and Ronald Pyke, Doubly stochastic operators obtained from

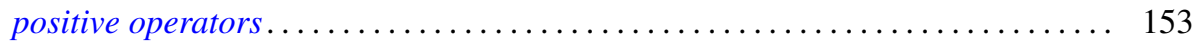

Robert Franklin Jolly, Concerning periodic subadditive functions . . . . . . . . . 159

Tosio Kato, Wave operators and unitary equivalence . . . . . . . . . . . . . . 171

Paul Katz and Ernst Gabor Straus, Infinite sums in algebraic structures . . . . . . . 181

Herbert Frederick Kreimer, Jr., On an extension of the Picard-Vessiot theory ...... 191

Radha Govinda Laha and Eugene Lukacs, On a linear form whose distribution is

identical with that of a monomial ......................... 207

Donald A. Ludwig, Singularities of superpositions of distributions . . . . . . . . . 215

Albert W. Marshall and Ingram Olkin, Norms and inequalities for condition

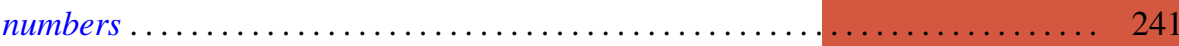

Horace Yomishi Mochizuki, Finitistic global dimension for rings . . . . . . . . . . 249

Robert Harvey Oehmke and Reuben Sandler, The collineation groups of division

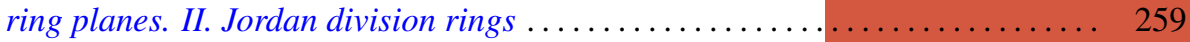

George H. Orland, On non-convex polyhedral surfaces in $E^{3} \ldots \ldots \ldots \ldots \ldots \ldots \ldots 267$

Theodore G. Ostrom, Collineation groups of semi-translation planes . . . . . . . . 273

Arthur Argyle Sagle, On anti-commutative algebras and general Lie triple

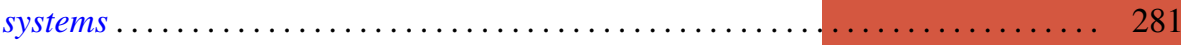

Laurent Siebenmann, A characterization of free projective planes . . . . . . . . . 293

Edward Silverman, Simple areas.................................. 299

James McLean Sloss, Chebyshev approximation to zero .................. 305

Robert S. Strichartz, Isometric isomorphisms of measure algebras . . . . . . . . . 315

Richard Joseph Turyn, Character sums and difference sets . . . . . . . . . . . . 319

L. E. Ward, Concerning Koch's theorem on the existence of arcs . . . . . . . . . . 347

Israel Zuckerman, A new measure of a partial differential field extension ......... 357 